\title{
Neumonía grave del adulto adquirida en la comunidad
}

FRANCISCO ARANCIBIA H. y ORLANDO DÍAZ P.

Severe community-acquired pneumonia in adults

Patients with severe community acquired pneumonia (CAP) need continuous surveillance and monitoring at intensive care units (ICU), where they can receive specialized support as mechanical ventilation and/or hemodynamic support. Patients that require ICU admittance represent 10 to $30 \%$ of all patients interned because a pneumonia. In this category, high complication rate, prolonged hospital stay and high mortality rate are the rule. The American Thoracic Society (ATS) criteria for severe pneumonia establishes the following main criteria: necessity of mechanical ventilation and presence of septic shock; minor criteria: systolic blood pressure $<90 \mathrm{mmHg}$, radiological multilobar involvement and $\mathrm{PaO}_{2} / \mathrm{FiO}_{2}<250 \mathrm{mmHg}$. British Thoracic Society (BTS) criteria for severe CAP are: respiratory rate over 30 breaths/min, diastolic blood pressure under $60 \mathrm{mmHg}$, BUN $>20 \mathrm{mg} / \mathrm{dl}$ and mental confusion. In all patients with CAP it is recommended the evaluation of its severity at admission. This evaluation should be done in conjunction with an experienced physician, and if criteria for poor prognosis are met, an early admission to ICU is recommended. ATS and BTS modified criteria (CURB) are useful in this procedure. In severely ill patients with CAP it is recommended to perform the following microbiological analysis: sputum Gram stain and culture, blood culture, pleural fluid Gram stain and culture, if present and tapped, Legionella pneumophila urine antigen test, influenza A and B antigen detection tests (epidemic period: autumn and winter), and serology for atypical bacteria (Mycoplasma pneumoniae and Chlamydia pneumoniae).

Key words: pneumonia, diagnosis, respiratory failure, severe sepsis.

Palabras clave: neumonía, diagnóstico, falla respiratoria, sepsis grave.

\section{Introducción}

Las neumonías adquiridas en la comunidad (NAC) que requieren tratamiento en la Unidad de Cuidados Intensivos (UCI) representan entre el 10 y el $30 \%$ de los pacientes hospitalizados por NAC ${ }^{1,2}$. En los pacientes con NAC grave la tasa de complicaciones, estadía en el hospital y mortalidad son elevadas, variando esta última entre 21 y $54 \%$ según distintas series ${ }^{3}$. Por lo tanto, parece particularmente importante identificar a aquellos pacientes que tienen alto riesgo de complicaciones y muerte. La evaluación de la gravedad de la neumonía no sólo es crucial para la decisión de hospitalización y admisión en la UCI en forma precoz, sino también, para la solicitud de exámenes complementarios y la selección del tratamiento antibiótico empírico inicial.

\section{Definición de la neumonía comunitaria grave}

A pesar de la importancia que tiene determinar la gravedad del paciente con neumonía, no existe hasta ahora una definición exacta y universalmente aceptada de NAC grave. En la mayoría de los estudios, se ha definido como NAC grave a la población de pacientes que fueron admitidos a la UCI, una definición operacional retrospectiva ${ }^{1}$. Sin embargo, los criterios empleados para admitir a estos pacientes en la UCI aún no han sido claramente definidos, y estos pueden variar, dependiendo de la experiencia del médico y normativas del hospital. En general, los médicos tienden a subestimar la gravedad de estos pacientes ${ }^{4}$ [Ib].

Leeper y cols sugieren una definición operacional simple para la NAC grave, que estaría

Unidad de Cuidados Intensivos, Instituto Nacional del Tórax, Santiago, Chile (FAH).

Departamento de Enfermedades Respiratorias, Facultad de Medicina. Pontificia Universidad Católica de Chile,

Santiago, Chile (ODP). 
dada por la necesidad de vigilar y monitorear al paciente en una UCI, permitiendo el apoyo con conexión a un ventilador mecánico y/o soporte hemodinámico ${ }^{5}$. En un intento por lograr una mejor definición de NAC grave, se han desarrollado varios sistemas de puntaje y modelos predictores para ayudar al médico clínico a identificar precozmente a estos pacientes. Sin embargo, ninguno de ellos como veremos a continuación, ha logrado una segura categorización del riesgo particular de cada paciente .

En 1987, la Sociedad Británica de Tórax (British Thoracic Society-BTS) desarrolló una regla predictora que intenta pronosticar el riesgo de muerte del paciente con neumonía basado en la medición de tres parámetros clínicos o criterios pronósticos: frecuencia respiratoria $>30$ respiraciones/min, presión arterial diastólica $<60$ $\mathrm{mmHg}$ y nitrógeno ureico $>20 \mathrm{mg} / \mathrm{dl}$ en la admisión al hospital ${ }^{7}$ [Ib]. Los pacientes que cumplían dos de estos tres criterios presentaban 21 veces mayor riesgo de muerte. Esta regla predictora que ha sido validada en otras poblaciones, presenta una sensibilidad de $88 \%$ y especificidad de $79 \%$, pero ha mostrado un bajo valor predictor positivo de $19 \%$.

Posteriormente, a los tres criterios originales se adicionó la presencia de confusión mental de reciente comienzo ${ }^{8}$. Para recordar mejor estos criterios pronósticos, se adoptó la regla nemotécnica inglesa CURB: confusion, urea, respiratory rate, and blood pressure ${ }^{9}$. La presencia de dos o más de estos cuatro criterios clínicos predice un mayor riesgo de muerte y debe ser considerado como un episodio de NAC grave. Así, en los pacientes que no tienen criterios de riesgo (CURB: 0) la mortalidad es cercana al $1 \%$, cuando tienen 1-2 criterios de riesgo la mortalidad es de $8 \%$ y cuando tienen 3-4 criterios la mortalidad asciende a $34 \%{ }^{10}$. En los estudios de validación, esta regla predictora tiene una sensibilidad de $83 \%$, especificidad de $70 \%$, y valor predictor positivo de sólo $26 \%$ [Ib]. Por otro lado, recientemente se ha comunicado que los criterios de la BTS serían menos sensibles en predecir la mortalidad de los pacientes mayores de 65 años 9

En 1993, la Sociedad Americana de Tórax (American Thoracic Society-ATS) publica las guías clínicas para el manejo de la $\mathrm{NAC}^{11}$. En ellas, se propone nueve criterios de gravedad para orientar la decisión de hospitalización en la UCI (Tabla 1). Cuando estos criterios fueron validados, en una población de pacientes hospitalizados con NAC, se encontró que la sensibilidad fue de $98 \%$ pero la especificidad de sólo
$32 \%{ }^{12}$. Es decir, los criterios son sensibles pero su bajo valor predictor positivo no permite discriminar qué pacientes requieren ser manejados en la UCI. Por este motivo, Ewig y cols proponen una nueva regla (criterios ATS modificados) que si bien disminuye la sensibilidad a $74 \%$, logra mejorar la especificidad a un $94 \%$ y el valor predictor positivo sube a $74 \%$ (Tabla 1$)^{12}$. Los criterios ATS modificados han sido validados recientemente en otro estudio ${ }^{11}$ [I Ib].

En un estudio multicéntrico, Fine y cols confeccionaron y validaron una nueva regla predictora denominada con la sigla PSI (pneumonia severity index $)^{13}$. Esta fue diseñada originalmente para identificar a pacientes con neumonía que tienen bajo riesgo de muerte, por tanto, que pueden ser tratados en forma ambulatoria. Posteriormente, el índice de gravedad de la neumonía (PSI) se ha utilizado en varios estudios para definir la neumonía grave. Sin embargo, se han observado discrepancias significativas al comparar los criterios convencionales de ingreso a la UCI y los puntajes de PSI. Por otro lado, en la práctica clínica ha sido complicado imple-

Tabla 1. Definición de neumonía comunitaria grave según la Sociedad Americana de Tórax y la Sociedad Británica de Tórax

Criterios originales de la Sociedad

Americana de Tórax

- Frecuencia respiratoria > 30 respiraciones/min (en la admisión)

- $\mathrm{PaO}_{2} / \mathrm{FiO}_{2}<250$ mmHg (en la admisión)

- Necesidad de ventilación mecánica

- Compromiso radiográfico bilateral o multilobar

- Aumento $\geq 50 \%$ infiltrados pulmonares en 48 horas

- Shock (PAS < 90 ó PAD < 60 mmHg)

- Necesidad de Fármacos vasoactivos $>4$ horas

- Diuresis $<20 \mathrm{ml} / \mathrm{h}$ o $<80 \mathrm{ml}$ en 4 horas

\section{Criterios modificados de la Sociedad Americana de Tórax \\ Criterios mayores (presencia de 1 criterio) \\ - Necesidad de ventilación mecánica \\ - Presencia de shock séptico}

Criterios menores (presencia de $\geq 2$ criterios)

- Presión sistólica $<90 \mathrm{mmHg}$

- Compromiso radiográfico multilobar

- $\mathrm{PaO}_{2} / \mathrm{FiO}_{2}<250 \mathrm{mmHg}$

Criterios de la Sociedad Británica de Tórax

- Frecuencia respiratoria $>30$ respiraciones/min

- Presión diastólica $<60 \mathrm{mmHg}$

- Nitrógeno ureico > $20 \mathrm{mg} / \mathrm{dl}$

- Confusión mental de reciente aparición

PAS = presión arterial sistólica; $\mathrm{PAD}=$ presión arterial diastólica 
mentar esta regla predictiva, dado que requiere recordar veinte criterios clínicos y de laboratorio para realizar los cálculos del puntaje, así parece ser poco práctica para el uso clínico rutinario. Además, el valor predictor de gravedad y riesgo de muerte es similar al instrumento más simple propuesto por la BTS (CURB) $)^{8,9}$.

El año 2001, la ATS ha actualizado las Guías Clínicas de NAC del adulto ${ }^{14}$, y ha definido la NAC grave por la presencia de los criterios de la ATS modificados ${ }^{12}$ o dos de los cuatro criterios de la BTS modificados ${ }^{8,9}$. Ninguna de estas definiciones es absolutamente sensible o específica.

\section{Recomendaciones}

Realizar, en todos los pacientes con NAC, una evaluación de la gravedad de la enfermedad al momento de su admisión al hospital ${ }^{6,14}$ [C]. Esta evaluación es preferible realizarla junto a un médico con experiencia, y si presenta criterios de mal pronóstico se sugiere trasladar precozmente a la $\mathrm{UCI}^{6}[\mathrm{D}]$. Son útiles para esta evaluación los criterios de la ATS modificados o los criterios de la BTS modificados (CURB). El juicio clínico y la experiencia del médico deben predominar sobre los modelos predictores, los cuales no son infalibles [D].

\section{Estudio microbiológico en la neumonía gra- ve adquirida en la comunidad}

En la NAC grave el rendimiento de los exámenes microbiológicos oscila entre un 48 y $72 \%$ según diferentes series ${ }^{15-18}$ (ver etiología, página 81 ). Se ha planteado realizar estudio microbiológico a los pacientes con NAC grave argumentando que esto permite confirmar el diagnóstico, identificar el agente causal de la NAC y conocer el patrón de resistencia a antimicrobianos de los microorganismos aislados en los cultivos, lo que permitiría ajustar el tratamiento antibiótico empírico inicial, ya sea modificándolo en caso de microorganismos resistentes, o realizar descalamiento o simplificación del esquema antibiótico, lo que tiene implicancias en el control de la resistencia bacteriana, contención de costos, reducción de los efectos adversos y por último por razones epidemiológicas. Recientemente, Rello y cols demostraron que el estudio microbiológico tiene algún impacto en el manejo antibiótico ${ }^{18}$. Este estudio fue realizado en una población de 204 pacientes con NAC grave, logrando identificar la etiología en el 57\% de los casos. La información microbiológica permitió simplificar el tratamiento en 65 pacientes (32\%) y modificarlo en 11 pacientes (5\%). Sin embargo, este y otros autores no han demostrado que la ejecución del estudio microbiológico logre modificar el pronóstico de los pacientes con NAC grave ${ }^{15,18,19}$ [II].

Hemocultivos: En la NAC grave el rendimiento de los hemocultivos es mayor y varía entre 14 y $27 \%$ según diversas series; sin embargo, disminuye en los pacientes que han utilizado antibióticos previamente ${ }^{16-18}$ [II]. Streptococcus pneumoniae continúa siendo el principal microorganismo aislado en los cultivos ${ }^{6,14}$ [II]. El hemocultivo positivo permite identificar el agente causal de la neumonía y tiene implicancias en el pronóstico. Fine y cols demostraron que la bacteriemia es un predictor independiente de mortalidad en pacientes con $\mathrm{NAC}^{20}$. Por tanto, es recomendable realizar dos hemocultivos aerobios antes de iniciar el tratamiento antibiótico empírico ${ }^{6,14}$ [B].

Cultivo de secreciones respiratorias: En todo paciente con NAC grave se debe intentar obtener una muestra de secreciones de la vía aérea inferior para su análisis. Si el paciente no está intubado, debe intentarse recolectar una muestra de esputo para tinción de Gram y cultivo corriente ${ }^{6,14}$ [D]. En pacientes intubados se recomienda efectuar tinción de Gram y cultivo cuantitativo del aspirado de secreción traqueobronquial precozmente ${ }^{6}$ [D]. Se considera significativo un nivel de corte $>10^{5} \mathrm{ufc} / \mathrm{ml}$. Sin embargo, no hay estudios en NAC que hayan evaluado el rendimiento de esta técnica comparado con las técnicas invasivas.

La búsqueda sistemática de tuberculosis aún es importante en nuestro medio, así se recomienda solicitar baciloscopias y cultivo de Koch en las muestras respiratorias obtenidas de los pacientes con NAC grave [D]. Debe realizarse precozmente en los pacientes con tos productiva persistente, baja de peso, sudoración nocturna o presencia de factores de riesgo para tuberculosis (origen étnico, deprivación social, alcoholismo, ancianos, inmunodeprimidos $)^{6}$ [D]. En algunas series, entre 1 y $2 \%$ de los pacientes con NAC corresponden a tuberculosis pulmonar asociado a insuficiencia respiratoria aguda, entre las cuales se debe recordar las formas de diseminación hematógena de la enfermedad (tuberculosis miliar).

Efusión pleural: En los pacientes que desarrollan una efusión pleural es obligatorio realizar análisis bioquímico, citológico y cultivo del líquido pleural para descartar un empiema ${ }^{6,14}[\mathbf{B}]$. 
Si se aisla algún microorganismo, esto confirma en forma definitiva la etiología de la neumonía y obliga al médico clínico a realizar un drenaje del espacio pleural.

Antígeno urinario: Se recomienda solicitar el antígeno urinario para Legionella pneumophila serogrupo 1 a todos los pacientes con NAC grave $^{6,14}[\mathbf{B}]$. Si bien, en Chile no existen estudios de prevalencia de L. pneumophila en este subgrupo de pacientes, estudios recientes han documentado la presencia de este agente, por lo que parece aconsejable realizarlo ${ }^{21,22}$.

Serología: Se recomienda realizar muestras pareadas para serología en todos los pacientes con NAC grave ${ }^{6,14}$ [D]. El incremento en los niveles de IgM de una muestra serológica simple, en fase aguda, también puede ser útil en el diagnóstico de neumonía por microorganismos atípicos (Mycoplasma pneumoniae, Chlamydia pneumoniae, L. pneumophila). No obstante, es menos sensible y específico al compararse con los resultados de la serología de muestras pareadas. En Chile, un estudio retrospectivo en pacientes con neumonía atípica y SDRA, en los cuales se descartó la infección por hantavirus, se encontró un $17,1 \%$ de serología positiva para M. pneumoniae y $8,6 \%$ para C. pneumoniae ${ }^{23}$.

En nuestro país esta técnica diagnóstica ha adquirido gran importancia en los últimos años para confirmar de forma rápida la sospecha clínica del síndrome cardiopulmonar por hantavirus $(\mathrm{SCPH})$ por cuanto tiene importantes repercusiones en el tratamiento ${ }^{24,25}$. El SCPH tiene una presentación estacional, concentrándose el mayor número de casos entre los meses de noviembre y marzo de cada año. Se han comunicado casos de SCPH desde la Región de Aconcagua hasta Aysén. Sin embargo, la XI ${ }^{\mathrm{a}}$ Región presenta el mayor riesgo, con una tasa de infección de 9 x 100.000 habitantes, seguido de la VIII ${ }^{\mathrm{a}}$ Región con 1 x 100.000 habitantes. En Chile, la letalidad de la infección pulmonar por hantavirus alcanza el 38\% (informe estadístico del Ministerio de Salud).

Detección de antígenos: En el período otoñoinvierno de brote de influenza también es recomendable realizar exámenes de detección rápida de virus influenza A y B, patógenos no siempre considerados en la etiología de la NAC grave [B]. La prueba rápida para detectar antígenos del virus influenza A o B en hisopado o aspirado nasofaríngeo permite establecer la etiología con sensibilidad y especificidad cercanas al $90 \%{ }^{26}$.
Técnicas invasoras: La fibrobroncoscopia no es recomendable realizarla en este subgrupo de pacientes si no están intubados, por cuanto existe un alto riesgo de hipoxemia y paro respiratorio, y no ha demostrado que modifique significativamente el manejo y pronóstico de los enfer$\operatorname{mos}^{6,19}$ [D]. La fibrobroncoscopia debe ser realizada después de la intubación del paciente con NAC grave en una UCI. Esta técnica puede ser muy útil para remover secreciones retenidas en la vía aérea, obtener muestras para cultivos $\mathrm{u}$ otras investigaciones microbiológicas, y descartar anormalidades endobronquiales, tales como un cuerpo extraño o neoplasia bronquial ${ }^{6}[\mathbf{C}]$.

La punción pulmonar percutánea y la punción transtraqueal son técnicas diagnósticas en desuso, ya que requerían personal calificado, tenían baja sensibilidad y elevadas tasas de complicaciones, en la actualidad no se recomienda realizarlas $^{6,27}$ [D]. Estas técnicas diagnósticas están contraindicadas en pacientes en ventilación mecánica.

Los cultivos de aspirado de secreciones traqueobronquiales obtenidas por el fibrobroncoscopio están contaminados con flora orofaríngea, determinando un rendimiento similar al cultivo de esputo. Esto hace necesario utilizar otras técnicas para la obtención de una muestra más representativa de las vías respiratorias bajas como son el cepillo protegido (CP) y el lavado broncoalveolar (LBA). El rendimiento diagnóstico del cepillo protegido en pacientes con NAC no intubados varía entre 54 y $85 \%$ de los $\operatorname{casos}^{27}$. En el subgrupo de pacientes con NAC grave conectados a ventilador mecánico, los cultivos son positivos en el 13 a $48 \%$ de los casos, dependiendo si han recibido antibióticos antes del procedimiento. Los cultivos cuantitativos obtenidos de LBA se correlacionan bien con el $\mathrm{CP}$ aunque con una menor sensibilidad ${ }^{27}$ (Tabla 2). El LBA obtiene una muestra respiratoria a partir de una mayor superficie pulmonar comparado con el CP y es particularmente útil en la

Tabla 2. Sensibilidad y especificidad de los cultivos bacterianos obtenidos por cepillo protegido (CP) y lavado broncoalveolar (LBA) en el diagnóstico etiológico de la neumonía comunitaria

\begin{tabular}{lcc}
\hline Procedimiento & Sensibilidad & Especificidad \\
\hline $\mathrm{CP}>10^{3} \mathrm{ufc} / \mathrm{ml}$ & $54-85 \%$ & $\geq 85 \%$ \\
$\mathrm{LBA}>10^{4} \mathrm{ufc} / \mathrm{ml}$ & $38-58 \%$ & $\geq 85 \%$ \\
\hline
\end{tabular}

Ref 27 
evaluación de pacientes inmunocomprometidos con infiltrados difusos en la radiografía de tórax. Sin embargo, estas técnicas diagnósticas tienen un bajo rendimiento en pacientes que han recibido tratamiento antibiótico antes del exa$\operatorname{men}^{27}$.

\section{Recomendaciones}

En concordancia con las guías clínicas de la $\operatorname{ATS}^{11,14}$ y BTS ${ }^{6}$, recomendamos realizar en los pacientes con NAC grave los siguientes exámenes microbiológicos: tinción de Gram y cultivo de expectoración, hemocultivos aerobios y cultivo de líquido pleural si hay efusión pleural puncionable, antígeno urinario para L. pneumophila, detección de antígenos de virus influenza A y B (durante el período epidémico de otoñoinvierno), y serología para gérmenes atípicos (M. pneumoniae y C. pneumoniae) [D].

\section{Resumen}

El paciente con neumonía grave adquirida en la comunidad es aquel que necesita de la vigilancia y monitorización de una Unidad de Cuidados Intensivos (UCI) donde, si es necesario, puede recibir apoyo especializado con conexión a un ventilador mecánico y/o soporte hemodinámico. Los pacientes que requieren tratamiento en la UCI representan entre 10 y $30 \%$ de los pacientes hospitalizados por neumonía. En esta categoría, la tasa de complicaciones, estadía en el hospital y mortalidad son elevadas. Los criterios para neumonía grave de la Sociedad Americana de Tórax (American Thoracic Society-ATS) son: criterios mayores: necesidad de ventilación mecánica y presencia de shock séptico; criterios menores: presión sistólica $<90 \mathrm{mmHg}$, compromiso radiográfico multilobar y $\mathrm{PaO}_{2} / \mathrm{FiO}_{2}<250 \mathrm{mmHg}$. Los criterios para NAC grave de la Sociedad Británica de Tórax (British Thoracic Society-BTS) son: frecuencia respiratoria mayor de 30 resp/min, presión diastólica menor de $60 \mathrm{mmHg}$, nitrógeno ureico $>20 \mathrm{mg} / \mathrm{dl}$ y confusión mental. En todos los pacientes con neumonía adquirida en la comunidad se recomienda evaluar la gravedad de la infección en el momento de su admisión al hospital. Esta evaluación es preferible realizarla junto a un médico con experiencia y, si presenta criterios de mal pronóstico, se sugiere trasladar precozmente a la UCI. Son útiles para esta evaluación los criterios de la ATS y los criterios de la BTS modificados (CURB). En los pacientes con neumonía grave adquirida en la comunidad se recomienda solicitar los siguientes exámenes microbiológicos: tinción de Gram y cultivo de expectoración, hemocultivos, tinción de Gram y cultivo de líquido pleural, antígeno urinario de Legionella pneumophila, antígenos de virus influenza A y B (período epidémico de otoño-invierno), y serología para microorganismos atípicos (Mycoplasma pneumoniae y Chlamydia pneumoniae).

\section{Bibliografía}

1.- Niederman M S. How do we optimize outcomes for patients with severe community-acquired pneumonia? Intensive Care Med 2002; 28: 1003-5.

2.- Angus D C, Marrie T J, Obrosky D S, Clermont G, Dremsizov T T, Coley C, et al. Severe communityacquired pneumonia: use of intensive care services and evaluation of American and British Thoracic Society Diagnostic criteria. Am J Respir Crit Care Med 2002; 166: 717-23.

3.- Ruíz M, Arancibia F, Torres A. Epidemiología, factores de riesgo y pronóstico de la neumonía grave adquirida en la comunidad. En Torres A, Mensa J, Niederman MS, eds. Infecciones Respiratorias en UCI. Editorial Springer-Verlag Ibérica 1999; pags. 1-12.

4.- Neill A M, Martín I R, Weir R, Anderson R, Chereshsky A, Epton M J, et al. Community acquired pneumonia: aetiology and usefulness of severity criteria on admission. Thorax 1996; 51: 1010-6.

5.- Leeper K V Jr, Torres A. Community-acquired pneumonia in the intensive care unit. Clin Chest Med 1995; 16: 155-71

6.- British Thoracic Society Standards of Care Committee. BTS Guidelines for the Management of Community Acquired Pneumonia in Adults. Thorax 2001; 56 (Suppl 4): iv1-iv64.

7.- British Thoracic Society and the Public Health Laboratory Service. Community-acquired pneumonia in adults in British hospitals in 1982-1983: a survey of aetiology, mortality, prognostic factors and outcome. Q J Med 1987; 62: 195-220.

8.- Karalus N C, Cursons R T, Leng R A, Mahood C B, Rothwell R P, Hancock B, et al. Community acquired pneumonia: aetiology and prognostic index evaluation. Thorax 1991; 46: 413-8.

9.- Lim W S, Macfarlane J T, Boswell T C, Harrison T G, Rose D, Leinonen M, Saikku P. Study of community acquired pneumonia aetiology (SCAPA) in adults admitted to hospital: implications for management guidelines. Thorax 2001; 56: 296-301.

10.- Ewing S, De Roux A, Bauer T, García E, Mensa J, Niederman M, Torres A. Validation of predictive rules and indices of severity for community acquired pneumonia. Thorax 2004; 59: 421-7.

11.- Niederman M S, Bass J B, Campbell G D, Fein A M, Grossman R F, Mandell L A, et al. Guidelines for the initial management of adults with community-acquired pneumonia: diagnosis, assessment of severity, and initial antimicrobial therapy. Am Rev Respir Dis 1993; 148:1418-26.

12.- Ewing S, Ruíz M, Mensa J, Marcos M A, Martínez J A, Arancibia F, et al. Severe community-acquired pneumonia. Assessment of severity criteria. Am J Respir Crit Care Med 1998; 158: 1102-8

13.- Fine M J, Auble T E, Yealy D M, Hanusa B H, Weissfeld L A, Singer D E, et al. A prediction rule to identify low-risk patients with community-acquired pneumonia. N Engl J Med 1997; 336: 243-50.

14.- Niederman M S, Mandell L A, Anzueto A, Bass J B, Broughton W A, Campbell G D, et al. Guidelines for the management of adults with community-acquired pneumonia. Diagnosis, assessment of severity, antimicrobial therapy, and prevention. Am J Respir Crit Care Med 2001; 163: 1730-54.

15.- Leroy O, Santre C, Beuscart C, Georges H, Guery B, Jacquier $\mathrm{J}$ M et al. A five-year study of severe community acquired pneumonia with emphasis on 
prognosis in patients admitted to an intensive care unit. Intensive Care Med 1995; 21: 24-31.

16.- Moine P, Vercken J B, Chevret S, Chastang C, Gajdos $\mathrm{P}$, and the French Study Group for CommunityAcquired Pneumonia in the Intensive Care Unit. Severe community-acquired pneumonia. Etiology, epidemiology, and prognosis factors. Chest 1994; 105: 1487-95.

17.- Ruíz M, Ewig S, Torres A, Arancibia F, Marco F, Mensa $\mathrm{J}$, et al. Severe community-acquired pneumonia. Risk factors and follow-up epidemiology. Am J Respir Crit Care Med 1999; 160: 923-9.

18.- Rello J, Bodi M, Mariscal D, Navarro M, Díaz E, Gallego M, et al. Microbiological testing and outcome of patients with severe community-acquired pneumonia. Chest 2003; 123: 174-80.

19.- Saldías F, Blacutt M, Moreno R. Manejo del paciente con neumonía grave en ventilación mecánica: utilidad del lavado broncoalveolar. Rev Méd Chile 1996; 124: 950-8.

20.- Fine M J, Smith M A, Carson C A, Mutha S S, Sankey S S, Weissfeld L A et al. Prognosis and outcomes of patients with community-acquired pneumonia. A metaanalysis. JAMA 1996; 275: 134-41.

21.- Trucco O, Vicencio M, Salamanca L, Ojeda A, Oyonarte M, Prado V. Participación de Legionella pneumophila en neumonía extrahospitalaria del adulto en Santiago. Rev Chil Infect 1993; 10: 8995.

22.- Cabello H, Cortés C, Ruíz M, Jover E, Rivera F, Segovia E, et al. Neumonía adquirida en la comunidad: comunicación de 8 casos de neumonía grave por Legionella pneumophila serogrupo 1 en Chile. Rev Méd Chile 2002; 130: 309-13.

23.- Navarrete M, Wilson M, Otth L, Hoffman I, Zaror L. Frecuencia de Mycoplasma pneumoniae y Chlamydia pneumoniae en pacientes con distress respiratorio y serología negativa para hantavirus. Rev Chil Infect 2003; 20: 7-10.

24.- Tapia M, Mansilla C, Vera J. Síndrome pulmonar por hantavirus: experiencia clínica en diagnóstico y tratamiento. Hospital Coyhaique-Chile. Rev Chil Infect 2000; 17: 258-69.

25. Castillo C, Naranjo J, Sepúlveda A, Ossa G, Levy H. Hantavirus pulmonary syndrome due to Andes virus in Temuco, Chile: clinical experience with 16 adults. Chest 2001; 120: 548-54.

26. Cox N J, Subbarao K. Influenza. Lancet 1999; 354: 1277-82.

27. Skerrett S J. Diagnostic testing for community-acquired pneumonia. Clin Chest Med 1999; 20: 531-48.

Correspondencia a:

Francisco Arancibia H.

franciscoarancibia@hotmail.com 